\title{
Sustained preventive chemotherapy for soil-transmitted helminthiases leads to reduction in prevalence and anthelminthic tablets required
}

Denise Mupfasoni ${ }^{*} \mathbb{D}$, Mathieu Bangert, Alexei Mikhailov, Chiara Marocco and Antonio Montresor

\begin{abstract}
Background: The goal of soil-transmitted helminthiases (STH) control programmes is to eliminate STH-associated morbidity in the target population by reducing the prevalence of moderate- and heavy-intensity infections and the overall STH infection prevalence mainly through preventive chemotherapy (PC) with either albendazole or mebendazole. Endemic countries should measure the success of their control programmes through regular epidemiological assessments. We evaluated changes in STH prevalence in countries that conducted effective PC coverage for STH to guide changes in the frequency of PC rounds and the number of tablets needed.

Methods: We selected countries from World Health Organization (WHO)'s Preventive Chemotherapy and Transmission control (PCT) databank that conducted $\geq 5$ years of PC with effective coverage for school-age children (SAC) and extracted STH baseline and impact assessment data using the WHO Epidemiological Data Reporting Form, Ministry of Health reports and/or peer-reviewed publications. We used pooled and weighted means to plot the prevalence of infection with any STH and with each STH species at baseline and after $\geq 5$ years of PC with effective coverage. Finally, using the WHO STH decision tree, we estimated the reduction in the number of tablets needed.
\end{abstract}

Results: Fifteen countries in four WHO regions conducted annual or semi-annual rounds of PC for STH for 5 years or more and collected data before and after interventions. At baseline, the pooled prevalence was 48.9\% (33.1-64.7\%) for any STH, 23.2\% (13.7-32.7\%) for Ascaris lumbricoides, 21.01\% (9.7-32.3\%) for Trichuris trichiura and $18.2 \%$ (10.9-25.5\%) for hookworm infections, while after $\geq 5$ years of PC for STH, the prevalence was $14.3 \%(7.3-21.3 \%)$ for any STH, 6.9\% (1.3-12.5\%) for A. lumbricoides, 5.3\% (1.06-9.6\%) for T. trichiura and $8.1 \%(4.0-12.2 \%)$ for hookworm infections.

Conclusions: Countries endemic for STH have made tremendous progress in reducing STH-associated morbidity, but very few countries have data to demonstrate that progress. In this study, the data show that nine countries should adapt their PC strategies and the frequency of PC rounds to yield a 36\% reduction in drug needs. The study also highlights the importance of impact assessment surveys to adapt control strategies according to STH prevalence.

Keywords: Soil-transmitted helminthiases, Control; morbidity, Preventive chemotherapy, Prevalence

\footnotetext{
* Correspondence: mupfasonid@who.int

Department of Control of Neglected Tropical Diseases, World Health

Organization, Geneva, Switzerland
}

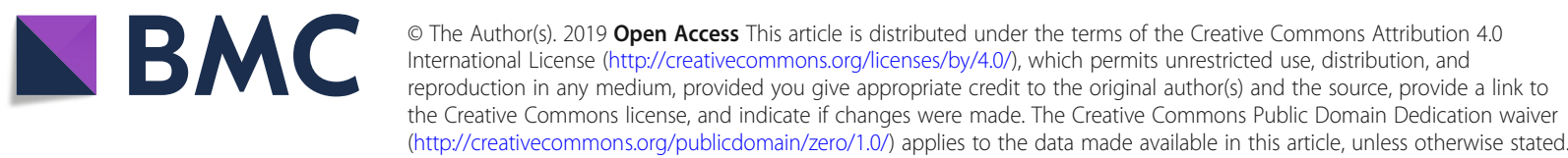




\section{Multilingual abstracts}

Please see Additional file 1 for translations of the abstract into the five official working languages of the United Nations.

\section{Background}

Soil-transmitted helminths are a group of intestinal parasites that are transmitted to humans through ingestion of infective eggs or transcutaneous penetration of larvae excreted in human faeces which contaminate the soil and water sources [1]. Soil-transmitted helminthiases (STH) are among the most common neglected tropical diseases (NTDs) in developing countries. Worldwide, it is estimated that 820 million people are infected with roundworm (Ascaris lumbricoides), 460 million with whipworm (Trichuris trichiura) and 460 million with hookworms (Necator americanus and Ancylostoma duodenale) in 102 countries [2]. In 2010, these infections contributed 3.4 million disability-adjusted life-years to the global burden of disease [1].

The World Health Organization (WHO) recommends preventive chemotherapy (PC) with albendazole or mebendazole to control STH-related morbidity alongside targeted health education and improved water and sanitation [3]. PC is the regular, coordinated administration of anthelminthic medicines to population groups at risk for STH morbidity. The target populations include preschool-age children (PSAC), school-age children (SAC), women of reproductive age (WRA) and adult groups particularly exposed to STH. The $2020 \mathrm{WHO}$ goal for control of STH is to treat $\geq 75 \%$ of PSAC and SAC in all STH endemic countries [4]. In 2017, more than 500 million SAC (69\% of total SAC in need) received PC for STH globally, with $73 \%$ of implementation units reaching $75 \%$ effective coverage [5].

The main objective of the STH control programme is to eliminate morbidity in the target population by reducing the prevalence of moderate- and heavy-intensity infections to $<2 \%$ [3]. A meta-analysis conducted by Marocco et al. showed that after five years of PC, $80 \%$ of individuals with infections of moderate and heavy intensity were cured or remained with only light-intensity infection [6].

Since 2010, WHO with the support of drug producers has donated albendazole or mebendazole for the control of STH. In 2018 alone, more than 485 million tablets were donated to endemic countries [5].

The WHO manual on STH control in SAC recommends that control programmes managers collect parasitological indicators every 3-5 years of PC with effective coverage in order to measure the effect of the intervention on the health of the population at risk [4] and, eventually, to reduce the number of $\mathrm{PC}$ rounds if the prevalence of $\mathrm{STH}$ infection has reduced to a certain level using the WHO decision tree (Additional file 2) [4].
In this study we evaluated the changes in $\mathrm{STH}$ prevalence from countries that conducted STH PC for $\geq 5$ years with effective treatment coverage (that is, national treatment coverage $\geq 75 \%$ as defined by WHO) for SAC. These data also give an indication of expected changes in prevalence if countries conduct $\geq 5$ years of $\mathrm{PC}$, and therefore also the expected number of drugs needed by endemic countries over time.

\section{Methods}

\section{National PC coverage data}

We accessed WHO's Preventive Chemotherapy and Transmission control (PCT) databank, an open webbased database on PC implementation in 102 endemic countries [7], to identify countries that had implemented effective coverage of $\mathrm{PC}$ (defined as $\geq 75 \%$ national coverage) among $\mathrm{SAC}$ for $\geq 5$ years.

\section{Epidemiological data reporting form}

The Epidemiological Data Reporting Form (EPIRF) is a standardized form designed to collect epidemiological data on all diseases targeted by PC, namely STH, lymphatic filariasis (LF), onchocerciasis and schistosomiasis. Indicators reported include: survey type, number of rounds of PC delivered prior to survey, date of survey, number of people examined, and numbers of people diagnosed positive for each STH species and for overall STH. Countries receiving donated anthelminthic medicines are invited to periodically report to WHO any changes in the epidemiological situation using the EPIRF. We extracted baseline and impact EPIRF data from endemic countries previously identified as having achieved $\geq 5$ years of effective coverage.

\section{Literature search}

For countries in which the EPIRF was not available we reviewed the available literature on baseline and impact assessment surveys for STH prevalence. Only surveys targeting SAC were considered. We searched official publications such as national NTD master plans and Ministry of Health survey reports as well as data published in the peer-reviewed scientific literature between 2000 and 2017. For online searches we used the words: mapping, baseline, impact assessment, soil-transmitted helminths and country names using google search and MEDLINE.

\section{Data analysis}

Studies were eligible for inclusion if, for baseline, the data were collected before the national STH control programme was initiated and, for impact assessment, the data were collected after PC was implemented for $\geq 5$ years with effective coverage. In addition, for all studies, SAC was the study population, the information on STH 
prevalence overall and/or for each STH species available. From each publication identified we extracted year, type of survey, target population, prevalence of STH and of each species, number of people examined, intensity of STH infection and diagnostic tool used. For studies where prevalence was reported by species only, we calculated the prevalence of any STH infection using the following equation [4]:

$$
\frac{(\boldsymbol{a}+\boldsymbol{t}+\boldsymbol{h})-(\boldsymbol{a} * \boldsymbol{t}+\boldsymbol{a} * \boldsymbol{h}+\boldsymbol{t} * \boldsymbol{h})+(\boldsymbol{a} * \boldsymbol{t} * \boldsymbol{h})}{1.06}
$$

Where $a=$ prevalence of ascariasis; $t=$ prevalence of trichuriasis and $h=$ prevalence of hookworm infections (all expressed as a proportion).

Data were collated, cleaned, analysed and visualized using the R (Version 3.5, R core Team, Vienna, Austria) statistical program. For data in the EPIRF form, we estimated national means using weighted cluster data for each country and for each STH species. We plotted the prevalence of any STH infection and of each species before the start of PC for STH and the prevalence after $\geq 5$ years of PC with effective coverage using pooled and weighted means. Finally, according to the WHO STH decision tree (Additional file 2) and after $\geq 5$ years of PC with effective coverage, countries with STH prevalence $<2 \%$ should suspend PC, $<10 \%$ should conduct PC once every 2 years, and $<20 \%$ should do $\mathrm{PC}$ once a year. In this study, we also estimated the decrease in drug needs for countries according to the change in STH prevalence during the $\geq 5$ years of PC.

\section{Results}

Number of countries that conducted $\geq 5$ years of PC for STH From the WHO/PCT databank we identified 24 countries that conducted $\geq 5$ years of PC for STH (Additional file 3) between 2003 and 2017. Three countries (Burkina Faso, Mali and Togo) reported their baseline data and 11 countries (Burkina Faso, Burundi, Mali, Ghana, Sierra Leone, Cameroon, Rwanda, Mexico, Nicaragua, Bangladesh and Lao People's Democratic Republic) reported their impact assessment data through

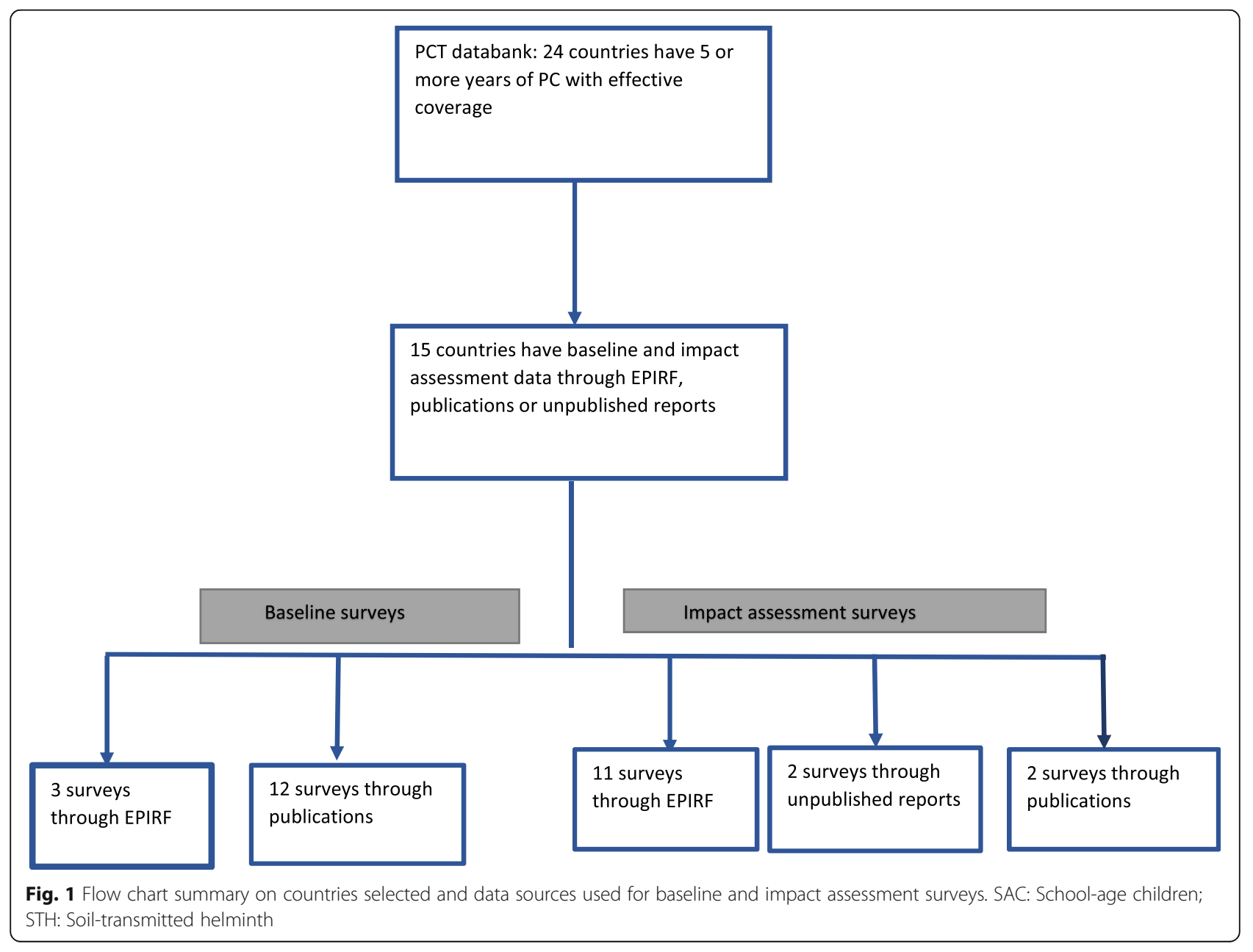




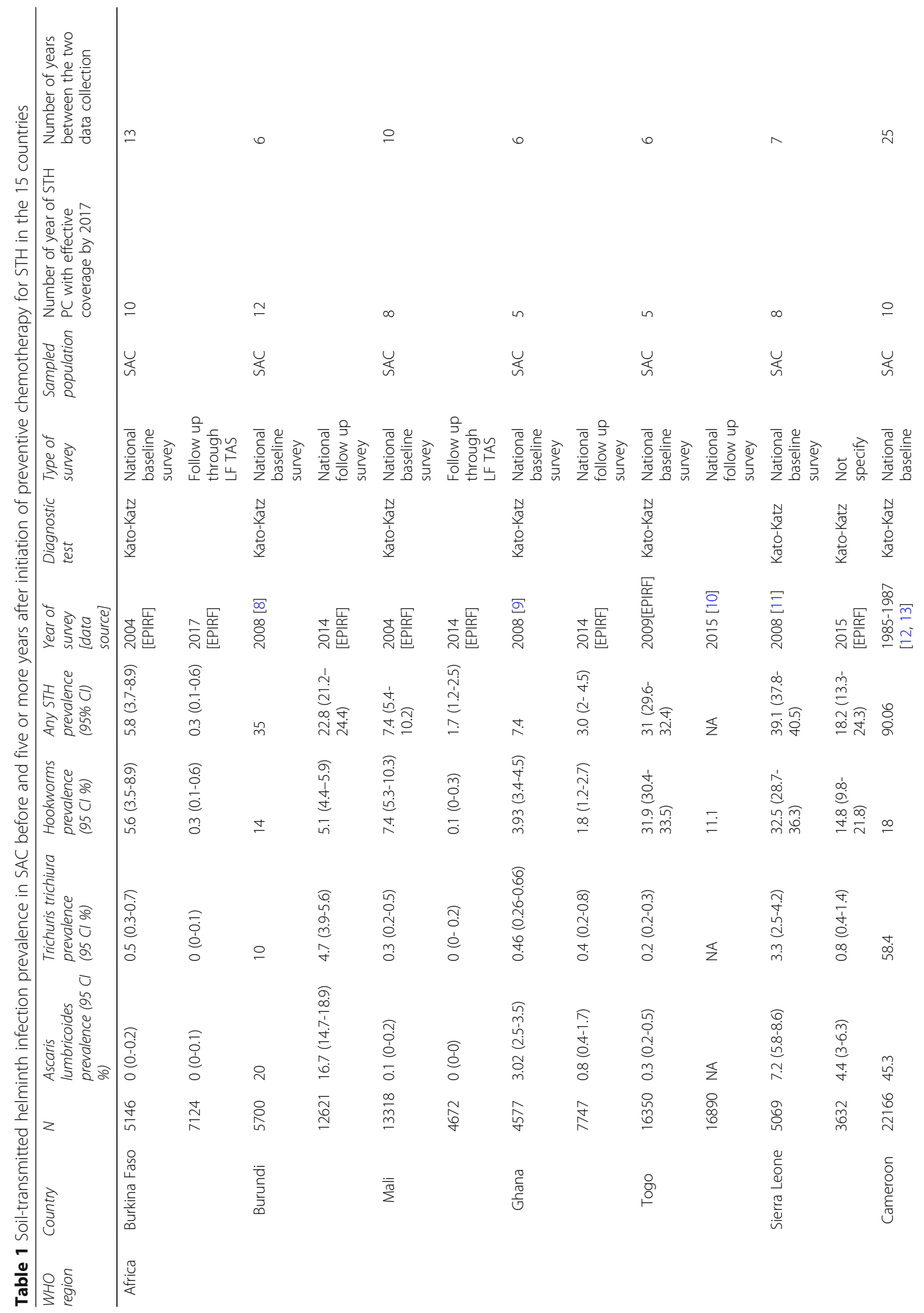




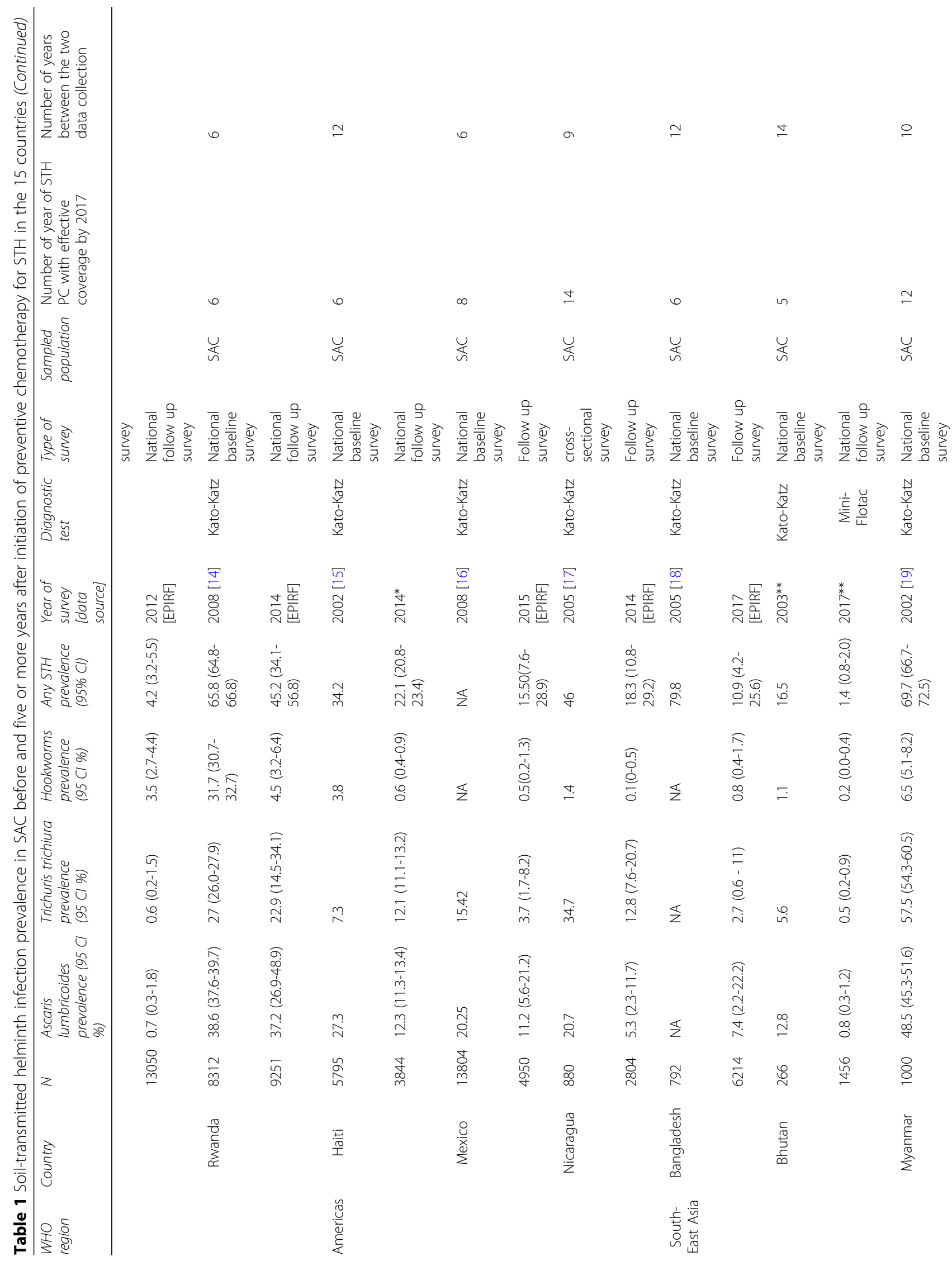




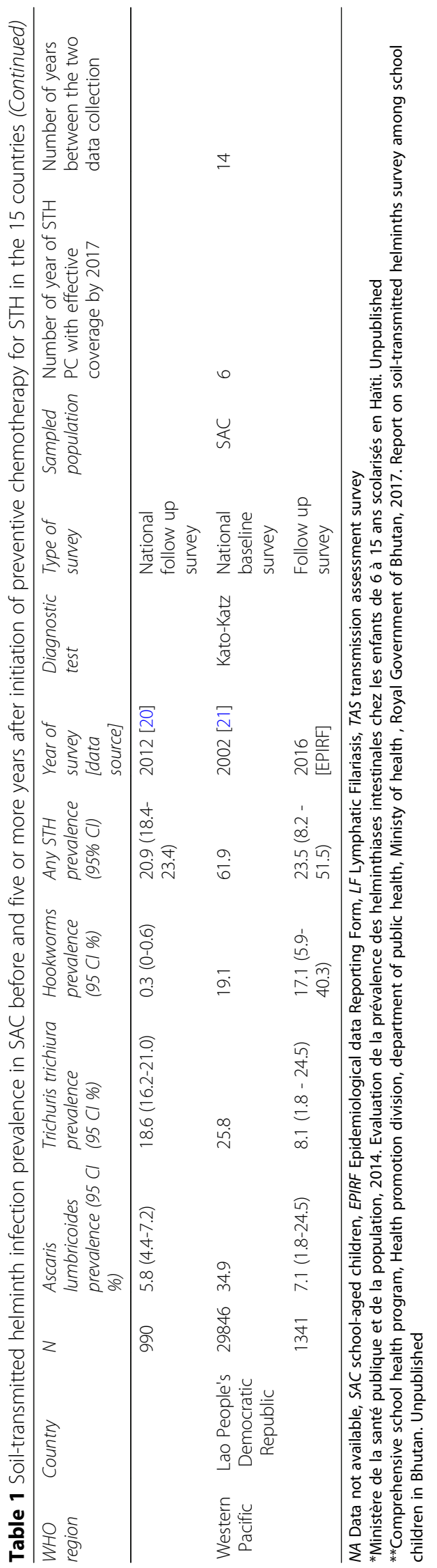




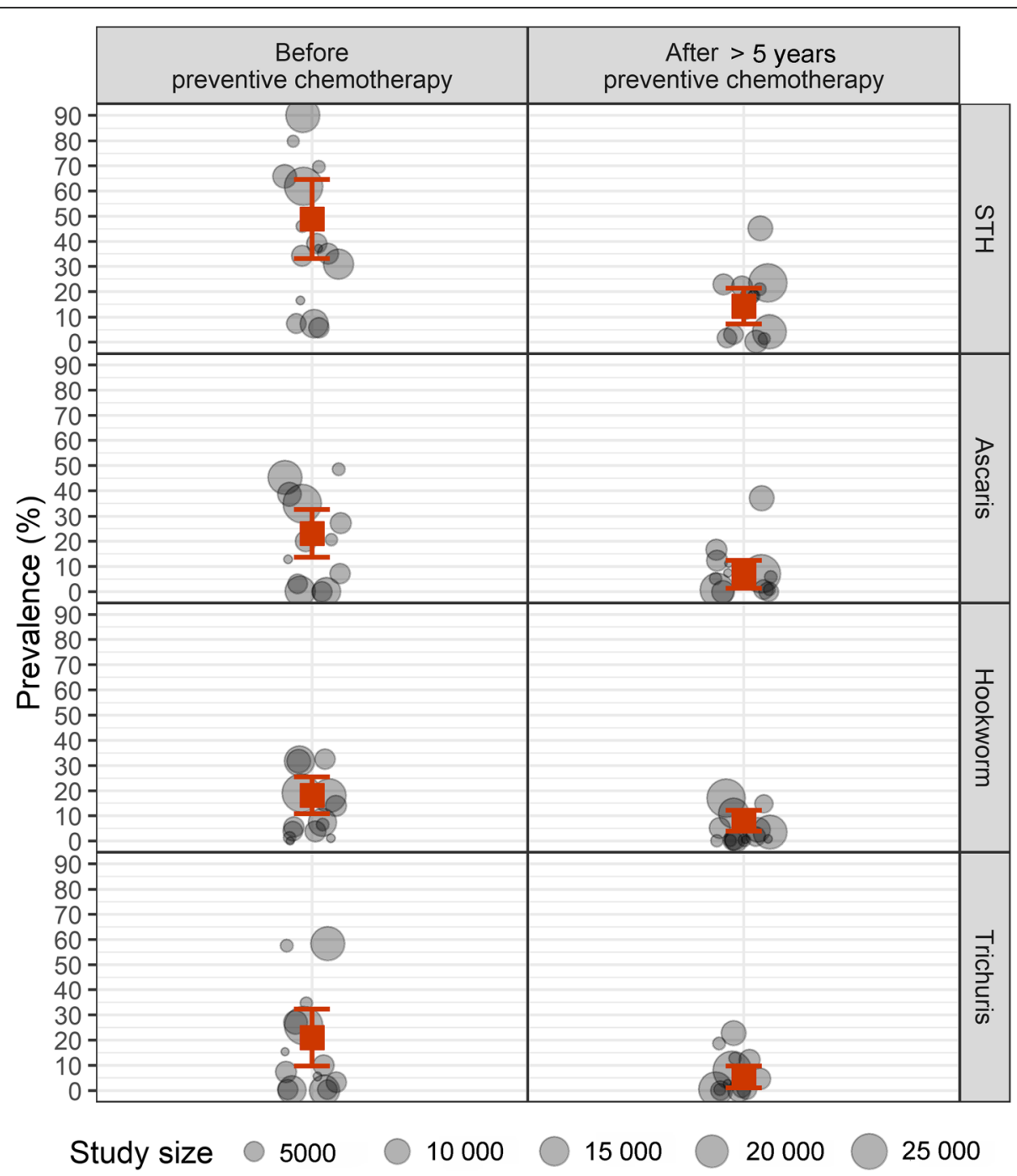

Fig. 2 Prevalence of STH (first row), Ascaris lumbricoides (second row), hookworm (third row) and Trichuris trichiura (fourth row) infections in countries before (left column) and after (right column) $\geq 5$ years of preventive chemotherapy with effective coverage. Each dot represents a published study or WHO EPIRF data. The size of the dot indicates the number of people assessed. The red square indicates pooled and weighted mean prevalence with 95\% confidence intervals. WHO: World Health Organization; EPIRF: Epidemiological data reporting form

EPIRF. Two countries (Burkina Faso and Mali) collected impact assessment data for STH through transmission assessment surveys (TAS) for LF. TAS is conducted after 5 years of annual LF MDA with a coverage over $65 \%$ to confirm that the prevalence of infection is below a threshold at which recrudescence is unlikely to occur. WHO recommends collecting data on STH epidemiology simultaneously in the area to determine if STH PC should be continued after LF MDA stopped. Ten countries conducted national surveys. In addition, we identified baseline surveys conducted before PC for 12 countries and identified two impact assessments surveys through publications and two unpublished reports on STH PC impact from the Ministry of Health. In total 15 countries in four WHO regions conducted PC STH for $\geq 5$ years and collected data before and after interventions (Fig. 1).

\section{Prevalence of STH infection at baseline before PC}

Most of the baseline data were collected during 20022009 (Table 1). The average size of the population surveyed was 8868 (range 266-22166) and $80 \%$ of the surveys were designed to be nationally representative. Six, four and four countries reported high, moderate and low STH prevalence, respectively. Figure 2 (left column) shows the pooled prevalence by country; the cumulative prevalence was $48.9 \%$ (33.1-64.7\%) for any STH, 23.2\% (13.732.7\%) for A. lumbricoides, $21.01 \%$ (9.7-32.3\%) for T. trichiura and $18.2 \%$ (10.9-25.5\%) for hookworm infections. 


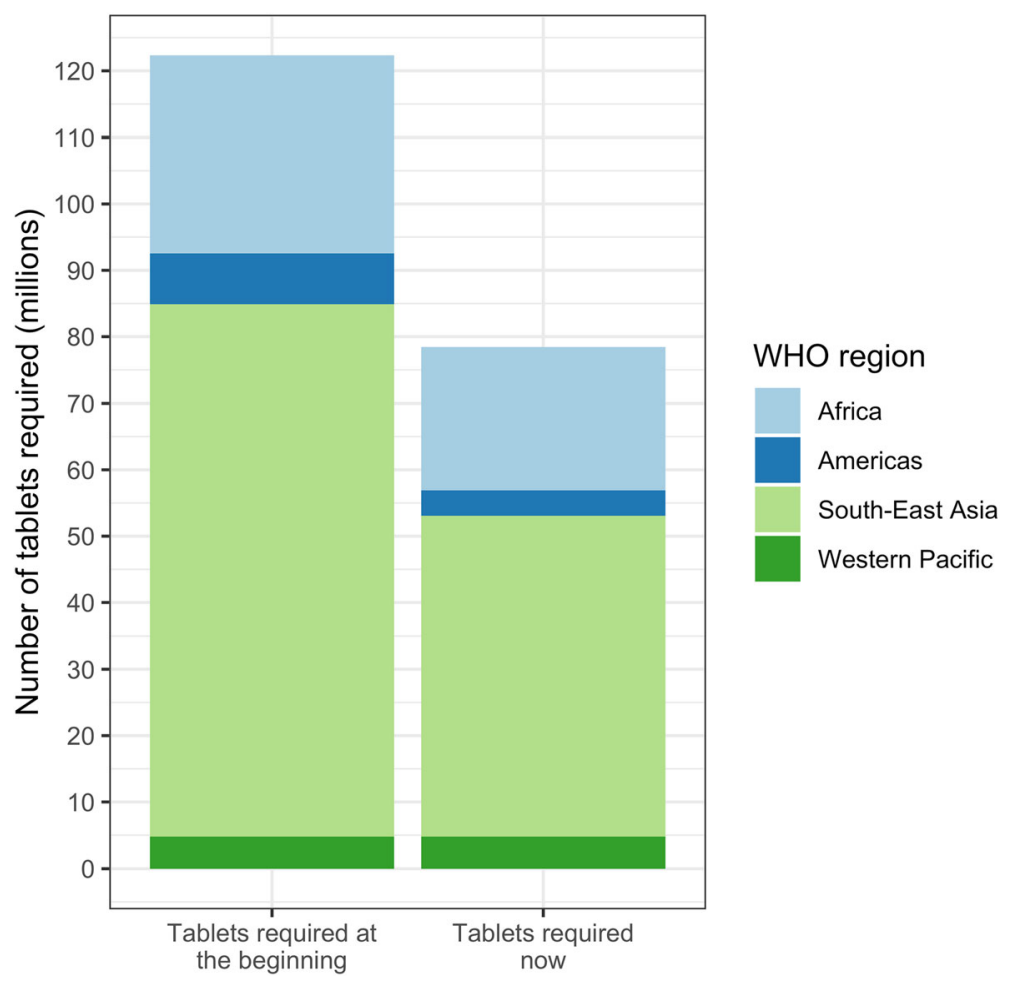

Fig. 3 Anthelminthic drug reduction after $\geq 5$ years of STH PC with effective coverage. STH: Soil-transmitted helminth; PC: Preventive chemotherapy

\section{Prevalence of STH infection after $\geq 5$ years of PC with effective coverage}

Between 2003 and 2017, the countries included in this study conducted and reported 5-14 rounds of PC, averaging eight rounds of PC for STH for SAC with effective coverage. Eleven countries conducted national surveys to measure the impact of PC on STH infection, while two countries collected the data during LF TAS (Table 1). These surveys were conducted during 2012-2017; the average population size surveyed was 6439 (range 99016 890). In five countries STH prevalence was moderate while in nine STH prevalence was low. The pooled prevalence was $14.3 \%(7.3-21.3 \%)$ for any $\mathrm{STH}, 6.9 \%$ (1.3-12.5\%) for A. lumbricoides, 5.3\% (1.06-9.6\%) for $T$. trichiura and $8.1 \%(4.0-12.2 \%)$ for hookworm infections (Fig. 2, right column). In countries with low-level STH prevalence at the beginning of their STH control programme, the prevalence was reduced to $<2 \%$ while countries with high prevalence moved to low or moderate prevalence (Table 1$)$.

\section{Estimated reduction in number of anthelminthic tablets}

For the 5 years and more of PC for STH, five countries conducted one round annually while 10 countries did semi-annual rounds. Figure 3 shows that if the 15 countries that conducted PC for $\geq 5$ years had applied WHO thresholds for changing the frequency of PC rounds using the impact assessment results on STH infection, the number of anthelminthic tablets needed for STH PC would have reduced by an average of $36 \%$. According to the WHO decision tree (Additional file 2), three countries would have suspended STH PC in some areas (because the STH prevalence was $<2 \%$ ) while six would have decreased the frequency of PC from semi-annual to annual or biennial, and six countries would have maintained the previous frequency of PC.

\section{Discussion}

In this study we report data from $15 \mathrm{STH}$ endemic countries that conducted $\geq 5$ years of PC with effective coverage among SAC. The overall result demonstrates a reduction of any STH prevalence and of STH species prevalence overall, representing tremendous progress for these countries. Importantly, in three countries the prevalence of any STH in the follow up survey was less than $2 \%$, meaning that they successfully eliminated STH morbidity.

A change in prevalence status implies a change in the frequency of PC rounds according to the WHO decision tree (Additional file 2). Countries with an STH prevalence $<2 \%$ after $\geq 5$ years of PC should suspend the intervention and maintain surveillance to detect possible rebounds of prevalence, whereas countries with a prevalence of $2-10 \%$ should proceed to semi-annual PC. To maintain the gains 
made when scaling down or discontinuing PC for $\mathrm{STH}$, the national STH control programme should ensure that the water, sanitation and hygiene (WASH) component is also implemented. A modelling study on the impact of deworming and WASH on STH transmission has shown that discontinuing $\mathrm{PC}$ and sustaining $\mathrm{PC}$ gains require continuation of WASH interventions [22]. Only by achieving Sustainable Development Goal 6, which seeks to ensure universal access to basic WASH in communities, schools and healthcare facilities by 2030 , will reductions of morbidity due to STH infection and others NTDs associated with water and sanitation be sustained [23].

Among the 15 countries included in the study, 10 are co-endemic for LF so community-based deworming was the strategy used. Most of these countries have already started scaling down mass drug administration (MDA) for LF. Three countries (Burkina Faso, Mali and Ghana) had a low STH prevalence at the beginning of the programme, but by conducting LF MDA with ivermectin and albendazole or diethylcarbamazine citrate and albendazole, STH infections were also treated. Meanwhile, in 2019, many implementation units have already stopped PC for LF and STH.

The limitations of this study are that the data used as the baseline for STH prevalence may have been collected after the start of LF elimination programmes and thus may under-evaluate the real STH prevalence before mass treatment was initiated. For countries in which we know this was true, we used survey data collected before the start of MDA for LF, as in Cameroon, where we used baseline data collected in 1987. Moreover, impact assessment surveys were never implemented in individuals who had participated also in the baseline surveys, sometimes not even in the same area, making the comparison difficult. Finally, not all surveys were nationally representative and thus did not truly reflect the status of STH endemicity in the country. Standardized implementation of impact surveys in all endemic countries would strengthen these data.

\section{Conclusions}

This study shows the importance of conducting impact assessment surveys after $\geq 5$ years of STH PC with effective coverage to guide national STH control programmes in adapting the number of PC rounds according to the new epidemiological situation. Furthermore, a reduction in the frequency of $\mathrm{PC}$ and the consequent reduction of associated costs would help endemic countries to achieve national support for, and ownership of, their PC programmes. It would be necessary to confirm that, in different countries and in different epidemiological situation, the reduction in frequency of PC suggested by the decision tree is sufficient to maintain the improvement achieved.

\section{Additional files}

Additional file 1: Multilingual abstracts in the five official working languages of the United Nations. (PDF $496 \mathrm{~kb}$ )

Additional file 2: $\mathrm{WHO}$ decision tree for STH control programmes. (DOCX 178 kb)

Additional file 3: Soil-transmitted helminthiases (STH) preventive chemotherapy (PC) coverage, by country, 2003-2017. (DOCX 22 kb)

\section{Abbreviations \\ EPIRF: Epidemiological Data Reporting Form; LF: Lymphatic filariasis; MDA: Mass drug administration; NTD: Neglected tropical disease; PC: Preventive chemotherapy; PCT: Preventive chemotherapy and transmission control; PSAC: Preschool-age children; SAC: School-age children; STH: Soil-transmitted helminthiase; TAS: Transmission assessment surveys; WASH: Water, sanitation and hygiene; WHO: World Health Organization}

\section{Acknowledgements}

We are thankful for national NTD programme managers, WHO country and regional offices for providing the data through the WHO PCT databank. We also thank Karen CICERI-REYNOLDS and Albis Francesco GABRIELLI for proof reading the article.

\section{Authors' contributions}

$\mathrm{DM}, \mathrm{MB}$ and $\mathrm{AM}$ o conceptualized the study.DM and AM did data collection. $\mathrm{DM}$ and $\mathrm{MB}$ did data analysis. DM and MB drafted the full paper. AMo revised edited the manuscript. All authors contributed to final development of the article. All authors read and approved the final manuscript.

\section{Funding}

Not applicable.

\section{Availability of data and materials}

All relevant data are within the manuscript and its Additional files,

Ethics approval and consent to participate

Not applicable

\section{Consent for publication}

Not applicable

\section{Competing interests}

The authors declare that they have no competing interests.

Received: 28 May 2019 Accepted: 14 August 2019

Published online: 02 October 2019

References

1. Montresor A, Trouleau W, Mupfasoni D, Bangert M, Joseph SA, Mikhailov A, Fitzpatrick C. Preventive chemotherapy to control soil-transmitted helminthiasis averted more than 500000 DALYs in 2015. Trans R Soc Trop Med Hyg. 2018. https://doi.org/10.1093/trstmh/trx082.

2. Guideline: preventive chemotherapy to control soil-transmitted helminth infections in at-risk population groups. Geneva: World Health Organization; 2017.https://apps.who.int/iris/bitstream/handle/10665/258983/978924155 0116-eng.pdf. Accessed 20 Mar 2019

3. Soil-transmitted helminthiases: eliminating soil-transmitted helminthiases as a public health problem in children: progress report 2001-2010 and strategic plan 2011-2020. Geneva: World Health Organization; 2012. https://apps.who.int/iris/bitstream/handle/10665/44804/9789241503129_ eng.pdf. Accessed 20 Mar 2019.

4. Helminth control in school-age children: a guide for managers of control programmes, 2nd. Geneva: World Health Organization; 2011. https://apps. who.int/iris/bitstream/handle/10665/44671/9789241548267_eng.pdf. Accessed 13 Mar 2019.

5. Schistosomiasis and soil-transmitted helminthiases: number of people treated in 2017. Wkly Epidemiol Rec. 2018:93:681-92 https://apps.who.int/ iris/bitstream/handle/10665/276933/WER9350.pdf. Accessed 15 Mar 2019. 
6. Marocco C, Bangert M, Joseph SA, Fitzpatrick C, Montresor A. Preventive chemotherapy in one year reduces by over $80 \%$ the number of individuals with soil-transmitted helminthiases causing morbidity: results from meta-analysis. Trans R Soc Trop Med Hyg. 2017. https://doi.org/10.1093/trstmh/trx011.

7. Yajima A, Mikhailov A, Mbabazi PS, Gabrielli AF, Minchiotti S, Montresor A, et al. Preventive chemotherapy and transmission control (PCT) databank: a tool for planning, implementation and monitoring of integrated preventive chemotherapy for control of neglected tropical diseases. Trans R Soc Trop Med Hyg. 2012;106:215-22.

8. Ortu G, Assoum M, Wittmann U, Knowles S, Clements M, Ndayishimiye O, et al. The impact of an 8-year mass drug administration programme on prevalence, intensity and co-infections of soil-transmitted helminthiases in Burundi. Parasit Vectors. 2016. https://doi.org/10.1186/s13071-016-1794-9.

9. Soares Magalhães RJ, Biritwum N-K, Gyapong JO, Brooker S, Zhang Y, Blair L, et al. Mapping helminth co-infection and co-intensity: geostatistical prediction in Ghana. PLoS Negl Trop Dis. 2011. https://doi.org/10.1371/journal.pntd.0001200.

10. Baker JM, Trinies V, Bronzan RN, Dorkenoo AM, Garn JV, Sognikin S, et al. The associations between water and sanitation and hookworm infection using cross-sectional data from Togo's national deworming program. PLoS Negl Trop Dis. 2018. https://doi.org/10.1371/journal.pntd.0006374.

11. Koroma JB, Peterson J, Gbakima AA, Nylander FE, Sahr F, Soares Magalhães RJ, et al. Geographical distribution of intestinal schistosomiasis and soiltransmitted helminthiasis and preventive chemotherapy strategies in Sierra Leone. PLoS Negl Trop Dis. 2010. https://doi.org/10.1371/journal.pntd.0000891.

12. Ratard RC, Kouemeni LE, Ekani Bessala MM, Ndamkou CN, Sama MT, Cline BL. Ascariasis and trichuriasis in Cameroon. Trans R Soc Trop Med Hyg. 1991. https://doi.org/10.1016/0035-9203(91)90170-4.

13. Ratard RC, Kouemeni LE, Ekani Bessala MK, Ndamkou CN. Distribution of hookworm infection in Cameroon. Ann Trop Med Parasitol. 1992;86:413-8.

14. Mupfasoni D, Ruberanziza E, Karibushi B, Rujeni N, Kabanda G, Kabera M. National school prevalence survey on soil-transmitted helminths and schistosomiasis, Rwanda. Int J Antimicrob Agents. 2009;34(Suppl 2):S15.

15. Champetier de Ribes G, Fline M, Désormeaux AM, Eyma E, Montagut P, Champagne C, Pierre J, et al. Intestinal helminthiasis in school children in Haiti in 2002. Bull Soc Pathol Exot. 2005:98:127-32.

16. Flisser A, Valdespino JL, García-García L, Guzman C, Aguirre MT, Manon ML, et al. Using national health weeks to deliver deworming to children: lessons from Mexico. J Epidemiol Community Health. 2008. https://doi.org/10.1136/ jech.2007.066423.

17. Rosewell A, Robleto G, Rodríguez G, Barragne-Bigot P, Amador JJ, Aldighieri S. Soiltransmitted helminth infection and urbanization in 880 primary school children in Nicaragua, 2005. Trop Doc. 2010. https:/doi.org/10.1258/td.2010.090425.

18. A situation analysis: neglected tropical diseases in Bangladesh. Dhaka: Ministry of Health \& Family Welfare, Government of Bangladesh; 2010 http://pdf.usaid.gov/pdf_docs/pnady849.pdf. Accessed 15 Mar 2019.

19. Montresor A, Zin T, Padmasiri E, Allen H, Savioli L. Soil-transmitted helminthiasis in Myanmar and approximate costs for countrywide control. Tropical Med Int Health. 2004. https://doi.org/10.1111/j.1365-3156.2004.01297.

20. Tun A, Myat SM, Gabrielli AF, Montresor A. Control of soil-transmitted helminthiasis in Myanmar: results of 7 years of deworming. Tropical Med Int Health. 2013. https://doi.org/10.1111/tmi.12130

21. Rim HJ, Chai JY, Min DY, Cho SY, Eom KS, Hong SJ, et al. Prevalence of intestinal parasite infections on a national scale among primary schoolchildren in Laos. Parasitol Res. 2003. https://doi.org/10.1007/s00436-003-0963.

22. Coffeng LE, Vaz Nery S, Gray DJ, Bakker R, de Vlas SJ, Clements ACA. Predicted short and long-term impact of deworming and water, hygiene, and sanitation on transmission of soil-transmitted helminths. PLoS Negl Trop Dis. 2018. https://doi.org/10.1371/journal.pntd.0006758.

23. Bangert M, Molyneux DH, Lindsay SW, Fitzpatrick C, Engels D. The crosscutting contribution of the end of neglected tropical diseases to the sustainable development goals. Infect Dis Poverty. 2017. https://doi.org/1 0.1186/s40249-017-0288-0.

Ready to submit your research? Choose BMC and benefit from:

- fast, convenient online submission

- thorough peer review by experienced researchers in your field

- rapid publication on acceptance

- support for research data, including large and complex data types

- gold Open Access which fosters wider collaboration and increased citations

- maximum visibility for your research: over $100 \mathrm{M}$ website views per year

At BMC, research is always in progress.

Learn more biomedcentral.com/submissions 